\title{
Evaluation of the Platelet Indices in Patients with Subclinical Hypothyroidism
}

\author{
Huseyin Avni Findikli ${ }^{1}$, Ayse Sahin Tutak ${ }^{2}$ \\ ${ }^{1}$ Department of Internal Medicine, Kahramanmaras Necip Fazil City Hospital, Turkey \\ ${ }^{2}$ Department of Internal Medicine, Adiyaman University, Turkey
}

"Corresponding Author: Huseyin Avni Findikli, Department of Internal Medicine, Kahramanmaras Necip Fazil City Hospital, Turkey, E-mail: dr-avni@hotmail.com; Tel: +90-3442282800

Received: 06 December 2018; Accepted: 14 December 2018; Published: 17 December 2018

\begin{abstract}
Objective: Both mean platelet volume (MPV) and platelet distribution width (PDW) can be used for various clinical results. The aim of this study was to establish a clinicopathological relationship by examining the thrombocyte parameters of subclinical hypothyroidism ( $\mathrm{SCH})$ cases.
\end{abstract}

Methods: A retrospective evaluation was made of 60 female subjects as a patient (subclinical) group of 30 females and an age-matched control group of 30 females.

Results: The mean age was similar in both study groups as $34.9 \pm 4.9$ years in the patient group and $33.8 \pm 3.19$ years in the control group ( $>0.05)$. The MPV and PDW values were measured higher in the patient than control group $(\mathrm{p}<0.05)$. TSH and platelet parameters, there was determined to be a correlation between MPV $(r=0.259, \mathrm{p}<0.05)$ and PDW $(\mathrm{r}=0.269, \mathrm{p}<0.05)$.

Conclusion: As a result, elevated MPV and PDW levels in patients with SCH may predispose for cardiovascular diseases.

Keywords: Subclinical hypothyroidism; Mean platelet volume; Platelet distribution width; Platelet indexes

\section{Introduction}

Subclinical hypothyroidism ( $\mathrm{SCH}$ ), which is defined as an elevated level of thyroid stimulating hormone (TSH) with the free thyroxin (fT4) level within the normal range, is the most common cause of Hashimoto thyroiditis. Prevalence varies between $1.3 \%$ and $17.5 \%$ depending on age, gender and iodine intake [1]. There is much evidence showing the relationship between $\mathrm{SCH}$ and cardiovascular diseases. Risk factors that can be determined in these patients include changes in the lipid 
profile, low-grade chronic inflammation, oxidative stress, diastolic hypertension, changes in coagulation parameters and impairments in the cardiovascular system [1-4].

Mean platelet volume (MPV) is the most commonly used measure of thrombocyte size and shows a correlation with thrombocyte activity. Platelet distribution width (PDW) is an index reflecting heterogenous thrombocyte size. These indexes, especially MPV and PDW, are related to thrombocyte functions $[5,6] . \mathrm{SCH}$ is a condition with a tendency to cardiac events. The aim of this study was to establish a clinicopathological relationship by examining the thrombocyte parameters of SCH cases.

\section{Subjects and Methods}

A retrospective examination was made of presented for treatment and follow-up at the Internal Diseases Polyclinic of Kahramanmaraş Necip Fazıl City Hospital between 01.06.2018 and 01.09.2018. Our study included 60 female subjects as a patient (subclinical) group of 30 females and an age-matched control group of 30 females. The patient group were diagnosed with subclinical hypothyrodism determined from TSH values higher than the reference intervals (0.27-4.2) and FT4 values within the reference intervals $(0.93-1.7 \mathrm{~g} / \mathrm{dL})$ In both groups, the voluntary participants had no chronic disease, biochemical and hematological laboratory results for electrolytes, fasting blood glucose, urea, creatinine, ferritin, B12 and folic acid were within the reference intervals. The biochemical and hematological laboratory test results data and the clinical results of the patients were obtained from the patient records of the hospital database.

\section{Statistical Analysis}

Data obtained in the study were analyzed statistically using SPSS Statistics vn 21 software (SPSS Inc., Chicago, IL, USA). Conformity with the data to normal distribution was assessed with visual (histogram and probability graphs) and analytical methods (Kolmogorov-Smirnov test). Variance homogeneity was evaluated with the Levene test. In descriptive analyses, variables with normal distribution were stated as mean \pm standard deviation (SD) values. For the difference between groups of parametric variables, the Student's t-test was applied. The cutoff values of independent significant predictors were analyzed with a Receiver Operating Characteristic (ROC) curve, and the area under the curve (AUC) was evaluated with the Hanley and McNeil method. A total type1 error level of 5\% was accepted as the level of statistical significance in all the statistical evaluations.

\section{Results}

The mean age was similar in both study groups as $34.9 \pm 4.9$ years in the patient group and $33.8 \pm 3.19$ years in the control group ( $\mathrm{p}>0.05)$. The basal demographic, clinical and laboratory data of both groups are shown in Table 1.

\begin{tabular}{|l|l|l|l|}
\hline Parameter & Controls $(\boldsymbol{n}=\mathbf{3 0})$ & Subclinic $(\boldsymbol{n}=\mathbf{3 0})$ & P value \\
\hline Age, years & $33.8 \pm 3.19$ & $34.9 \pm 4.9$ & 0.433 \\
\hline HGB, gr/dl & $13.2 \pm 0.63$ & $12.7 \pm 0.70$ & 0.080 \\
\hline WBC, 1000/ $\mathbf{u L}$ & $8.7 \pm 1.56$ & $8.5 \pm 1.54$ & 0.580 \\
\hline
\end{tabular}




\begin{tabular}{|l|l|l|l|}
\hline PC, 1000/ $\boldsymbol{\mu L}$ & $246 \pm 56.4$ & $237 \pm 64.5$ & 0.607 \\
\hline MPV, fL & $9.5 \pm 1.68$ & $10.7 \pm 2.04$ & $0.022^{*}$ \\
\hline PDW, fL & $11.4 \pm 1.69$ & $12.4 \pm 1.99$ & $0.032^{*}$ \\
\hline TSH & $2.11 \pm 1.04$ & $7.10 \pm 0.88$ & $<0.001^{*}$ \\
\hline FT4 & $0.92 \pm 0.19$ & $0.96 \pm 0.15$ & 0.398 \\
\hline
\end{tabular}

HGB-Hemoglobin; PC-Platellet Count; WBC-White Blood Cells; MPV-Mean Platelet Volume; PDW-Platelet Distribution Width; TSH-Thyroid-Stimulating Hormone; FT4-Free Thyroxine; *p-value is less than 0.05

Table 1: Demographic and biochemical characteristics of the study groups.

Significant differences were determined between the groups in respect of MPV and PDW values. The MPV and PDW values were measured higher in the patient than controls group $(\mathrm{p}<0.05)$ (Table 1, Figure 1).

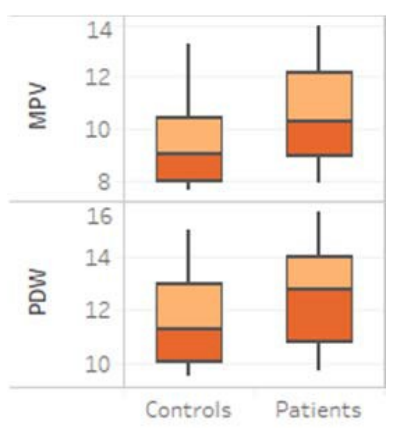

Figure 1: Comparison of Mean Platelet Volume (MPV) and Platelet Distribution Width (PDW) between the study groups.

In the correlation analysis made between TSH and platelet parameters, there was determined to be a correlation between MPV $(r=0.259, \mathrm{p}<0.05)$ and PDW $(r=0.269, \mathrm{p}<0.05)$ (Figure 2).

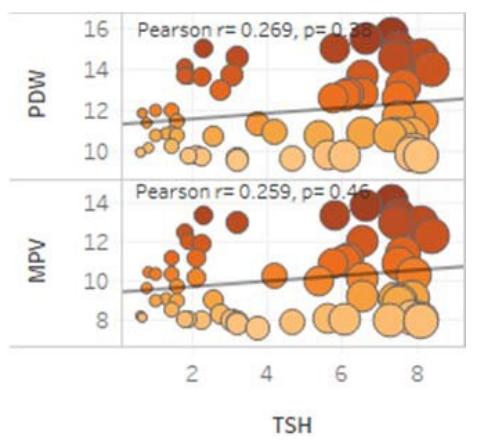

Figure 2: Correlation between Mean Platelet Volume (MPV) and Platelet Distribution Width (PDW) with TSH (Thyroid Stimulating Hormone) (Size shows details about TSH, color show details about MPV and PDW). 
AUCs of ROC analysis of MPV and PDW for the diagnosis of subclinic hypothyroidsm were $0.666(\mathrm{p}=0.028),(95 \% \mathrm{CI}$, $0.52-0.80)$ and $0.653(\mathrm{p}=0.041),(95 \% \mathrm{CI}, 0.51-0.79)$, respectively (Figure 3 ).

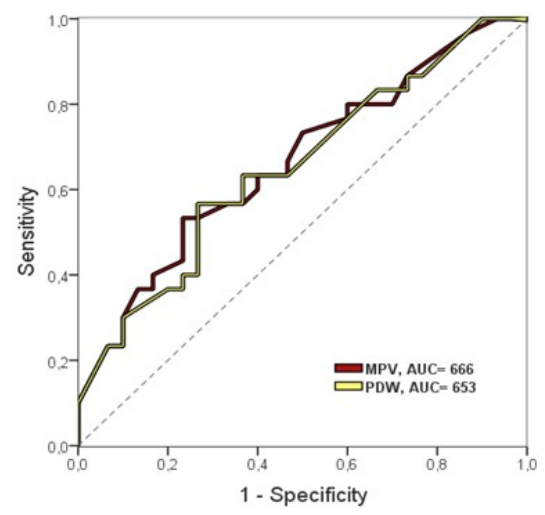

Figure 3: ROC curve with Area Under the Curve (AUC) for Mean Platelet Volume (MPV) and Platelet Distribution Width (PDW) predicting subclinical hypothyroidism.

The sensitivity decreased, whereas the specificity increased as the cutoff levels increased. The optimal MPV and PDW cutoff levels as identified by the maximal Youden index were 9.85 and for subclinic hypothyroidsm. In subclinic hypothyroidsm, the MPV cutoff of $6.0 \%$ and PDW showed high sensitivity (63.3\%) and specificity (60\%), with a low proportion (16.3\%) of false-negative results in disease identification.

\section{Discussion}

Subclinical hypothyroidism ( $\mathrm{SCH}$ ), which cannot be clearly categorized as different from hypothyroidism, tends to have a clinical course that is symptomatic and/or asymptomatic. Previous studies have reported that $\mathrm{SCH}$ is an independent risk factor for atherosclerosis and myocardial infarct and the mortality risk associated with cardiovascular causes has been reduced after treatment with levothyroxin at sufficient doses [3, 7-9]. In this context, the aim of this study was to investigate how the thrombocyte indexes are affected in $\mathrm{SCH}$ patients, as they are known to have a critical role in the pathogenesis and prognosis of acute coronary syndrome. The results of the study showed that the MPV and PLT values measured in the SCH group were significantly higher than those of the control group and the increase in these parameters had diagnostic value.

Platelet indexes are an index group used to measure PLT number and morphology. Under pathophysiological conditions, any factor which can inhibit PLT regeneration, increase activation or accelerate death by exceeding self-recovery capacity, can cause changes in both the number and morphology of PLT, and this therefore leads to changes in the PLT indexes [6, 10]. To date there have been few studies related to the change in platelet indexes in HT and SCH patients, and conflicting results have been reported.

Erikci et al. reported that MPV and PDW levels increased in HT and SCH patients, whereas there was no change in PLT levels. Coban et al. showed an increase in MPV values of SCH cases [11], Yilmaz, et al. reported that MPV levels 
increased but there was no change in PLT levels and Kim, et al. also showed an increase in MPV levels in SCH patients [12]. In contrast, Van Doormaal et al. stated that hypothyroidism led to smaller sized thrombocytes [13] and Ford, et al. also reported that chronic hypothyroidism resulted in smaller thrombocytes in circulation [14].

Similar to the findings of the four above-mentioned studies, the results of the current study showed that the MPV and PDW levels were higher in the SCH group than in the control group and the PLT levels were similar. The MPV and PLT levels were also seen to be correlated to the levels of TSH. These findings demonstrated that PLT indexes were useful in the prediction of SCH patients. To analyze the performance of PLT indexes for SCH patients, ROC analysis was applied to each PLT index and the diagnostic parameters were calculated for all the PLT indexes. The largest AUC values of ROC were found to be 0.78 for PLT and 0.79 for MPV. Therefore, it was concluded that MPV could be the optimal predictive marker for SCH patients.

Previous studies have shown that conditions such as coagulation system activation, severe infection, trauma, and systemic inflammatory reaction, could lead to changes in PLT indexes. A relationship has been determined between increased MPV and/or PDW values and various cardiovascular diseases $[9,15,16]$. The mechanism responsible for this is that larger thrombocytes are more active enzymatically or metabollically and thus there is high potential thrombotic activity [17]. As high-volume thrombocytes are more active hemostatically, they could be a risk factor for possible coronary thrombosis and development of myocardial infarct. The current study data of increased mean thrombocyte volume in SCH patients suggests that they could have a specific role in pre-thrombotic events that could develop in the future.

In conclusion, when it is accepted that increased MPV and PDW levels are risk factors for cardiovascular diseases, there is a need for further studies of larger patient cohorts with longer follow-up periods to be able to confirm the increased thrombocyte activation and associated increased risk of cardiovascular complications in $\mathrm{SCH}$ patients.

\section{Conflicts of Interest}

None

\section{References}

1. Biondi B, Palmieri EA, Lombardi G. EFfects of subclinical thyroid dysfunction on the heart. Annals of internal medicine 137 (2002): 904-914.

2. Tieche M, Lupi GA, Gutzwiller F, et al. Borderline low thyroid function and thyroid autoimmunity. Risk factors for coronary heart disease? British heart journal 46 (1981): 202-206.

3. Udovcic M, Pena RH, Patham B, et al. Hypothyroidism and the Heart. Methodist DeBakey cardiovascular journal 13 (2017): 55-59.

4. Biondi B. Cardiovascular effects of mild hypothyroidism. Thyroid: Official journal of the American Thyroid Association 17 (2007): 625-630.

5. Jackson SR, Carter JM. Platelet volume: laboratory measurement and clinical application. Blood reviews 7 (1993): 104-113. 
6. Zhang S, Cui Y-L, Diao M-Y, et al. Use of Platelet Indices for Determining Illness Severity and Predicting Prognosis in Critically Ill Patients. Chinese medical journal 128 (2015): 2012-2018.

7. Hak AE, Pols HA, Visser TJ, et al. Subclinical hypothyroidism is an independent risk factor for atherosclerosis and myocardial infarction in elderly women: the Rotterdam Study. Annals of internal medicine 132 (2000): 270278 .

8. Unal E, Akin A, Yildirim R, et al. Association of Subclinical Hypothyroidism with Dyslipidemia and Increased Carotid Intima-Media Thickness in Children. Journal of clinical research in pediatric endocrinology 9 (2017): 144-149.

9. Endler G, Klimesch A, Sunder-Plassmann H, et al. Mean platelet volume is an independent risk factor for myocardial infarction but not for coronary artery disease. British journal of haematology 117 (2002): 399-404.

10. Gado K, Domjan G. Thrombocytopenia. Orv Hetil 155 (2014): 291-303.

11. Coban E, Yazicioglu G, Ozdogan M. Platelet activation in subjects with subclinical hypothyroidism. Medical science monitor: International medical journal of experimental and clinical research 13 (2007): 211-214.

12. Yilmaz H, Ertugrul O, Ertugrul B, et al. Mean platelet volume in patients with subclinical hypothyroidism. Platelets 22 (2011): 143-147.

13. Van Doormaal JJ, Van der Meer J, Oosten HR, et al. Hypothyroidism leads to more small-sized platelets in circulation. Thrombosis and haemostasis 58 (1987): 964-965.

14. Ford HC, Carter JM. Haemostasis in hypothyroidism. Postgraduate medical journal 66 (1990): 280-284.

15. Kilicli-Camur N, Demirtunc R, Konuralp C, et al. Could mean platelet volume be a predictive marker for acute myocardial infarction? Medical science monitor: International medical journal of experimental and clinical research 11 (2005): 387-392.

16. Khandekar MM, Khurana AS, Deshmukh SD, et al. Platelet volume indices in patients with coronary artery disease and acute myocardial infarction: An Indian scenario. Journal of clinical pathology 59 (2006): 146-149.

17. Cesari F, Marcucci R, Caporale R, et al. Relationship between high platelet turnover and platelet function in highrisk patients with coronary artery disease on dual antiplatelet therapy. Thrombosis and haemostasis 99 (2008): 930-935.

Citation: Huseyin Avni Findikli, Ayse Sahin Tutak. Evaluation of the Platelet Indices in Patients with Subclinical Hypothyroidism. Archives of Clinical and Biomedical Research 2 (2018): 227-232.

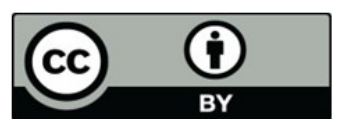

This article is an open access article distributed under the terms and conditions of the $\underline{\text { Creative Commons Attribution (CC-BY) license } 4.0}$ 